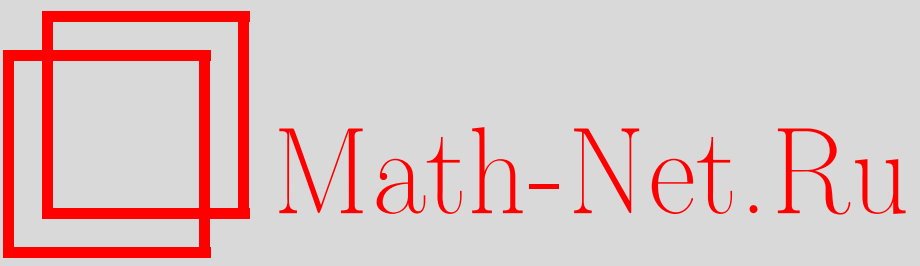

А. В. Цыганов, Канонические преобразования расширенного фазового пространства и интегрируемые системы, ТМФ, 2000, том 124, номер 1, 72-94

DOI: https://doi.org/10.4213/tmf627

Использование Общероссийского математического портала Math-Net.Ru подразумевает, что вы прочитали и согласны с пользовательским соглашением

http://www.mathnet.ru/rus/agreement

Параметры загрузки:

IP : 18.208 .226 .222

26 апреля 2023 г., 05:14:30 
ТЕОРЕТИЧЕСКАЯ

И МАТЕМАТИЧЕСКАЯ

ФИЗИКА

Том 124, № 1

июль, 2000

(C) 2000 г. $\quad$ А.В. Цыганов*

\section{КАНОНИЧЕСКИЕ ПРЕОБРАЗОВАНИЯ РАСШИРЕННОГО ФАЗОВОГО ПРОСТРАНСТВА И ИНТЕГРИРУЕМЫЕ СИСТЕМЫ}

Изучается вопрос о явном построении канонического преобразования переменной времени и гамильтониана, которое отображает данную вполне интегрируемую систему в другую интегрируемую систему. Замена времени индуцирует преобразование уравнений движения и их решений, интегралов движения и методов разделения переменных, матриц Лакса и соответствующих $r$-матриц. Для некоторых конкретных семейств интегрируемых систем (цепочки Тоды, системы Холта и системы типа Штеккеля) построены канонические преобразования времени в расширенном фазовом пространстве, сохраняющие свойство интегрируемости.

\section{1. ВВЕДЕНИЕ}

Изучение вполне интегрируемых систем классической механики остается одним из важнейших направлений исследований в математической физике. Под такими системами понимаются гамильтоновы системы с конечным числом степеней свободы, обладающие необходимым числом интегралов движения, так что решение уравнений движения может быть сведено к квадратурам.

Известно, что любые канонические преобразования координат фазового пространства $\mathcal{M}$ сохраняют свойство интегрируемости. Вопрос о том, какие общие преобразования, в том числе и неканонические, отображают данную вполне интегрируемую систему в другую интегрируемую систему, остается открытым.

В данной работе изучается класс обобщенных канонических преобразований расширенного фазового пространства $\mathcal{M}_{E}$, которые сохраняют свойство интегрируемости и являются композицией канонического преобразования переменной времени $t$ и сопряженного ей гамильтониана $H$ с каноническими преобразованиями остальных переменных.

В качестве примера канонических преобразований времени, сохраняюших интегрируемость, в данной работе построены новые интегрируемые системы, связанные с цепочками Тоды, системами Энона-Эйлеса и системами типа Штеккеля. Цепочки Тоды яв-

* Санкт-Петербургский государственный университет, Санкт-Петербург, Россия. E-mail:tsiganov@mph.niif.spb.su 
ляются естественным примером использования современных алгебро-геометрических методов в классической механике [1]. Поэтому соответствуюшая замена времени будет найдена с помощю алгебраических методов. В то же время системы типа Штеккеля были открыты в конце прошлого века в рамках метода разделения переменных [2]. Поэтому при поиске подходящей замены времени будет также использован метод разделения переменных

Хотя и некоторые цепочки Тоды, и системы типа Штеккеля связаны с гиперэллиптическими алгебраическими кривыми, природа предлагаемых для них преобразований времени различна. В силу этого работа состоит из двух частей, каждая из которых может быть рассмотрена независимо от другой. Мы сочли возможным объединить эти две части только для того, чтобы подчеркнуть разнообразие примеров канонических преобразований в расширенном фазовом пространстве и тем самым привлечь внимание к данной проблеме.

\section{2. КАНОНИЧЕСКИЕ ПРЕОБРАЗОВАНИЯ ВРЕМЕНИ В РАСШИРЕННОМ ФАЗОВОМ ПРОСТРАНСТВЕ}

Пусть на произвольном $2 n$-мерном симплектическом многообразии $\mathcal{M}$ с симплектической формой $\Omega$ задана некоторая гамильтонова система. По определению канонические преобразования фазового пространства $\mathcal{M}$ сохраняют канонический вид уравнений Гамильтона. Однако в уравнениях движения на фазовом пространстве $\mathcal{M}$ и поэтому в определении канонических преобразований пространства $\mathcal{M}$ время $t$ играет роль параметра, а не переменной.

Построим новое пространство $\mathcal{M}_{E}$, добавив к фазовому пространству $\mathcal{M}$ с координатами $\left\{p_{j}, q_{j}\right\}_{j=1}^{n}$ переменную времени $t=q_{n+1}$ вместе с сопряженной ей функцией Гамильтона $H=p_{n+1}$. Следуя работам $[3,4]$, далее будем называть $(2 n+2)$-мерное пространство $\mathcal{M}_{E}$ с координатами $(q, p, t, H)$ расширенным фазовым пространством. Определим канонические преобразования времени $t$ и гамильтониана $H$ как преобразования, которые сохраняют канонический вид уравнения Гамильтона-Якоби

$$
\frac{\partial S}{\partial t}+H=0
$$

Инвариантность уравнений движения относительно канонических преобразований приводит к инвариантности дифференциальной формы $\sum_{i=1}^{n+1} p_{i} d q_{i}$ [3]. Используя данные свойства инвариантности, определим каноническое преобразование переменной времени

$$
t \rightarrow \tilde{t}, \quad d \tilde{t}=v(p, q) d t
$$

и функции Гамильтона

$$
H \rightarrow \widetilde{H}=\frac{H}{v(p, q)}
$$

расширенного фазового пространства $\mathcal{M}_{E}$.

Канонические преобразования расширенного фазового пространства определены для любой гамильтоновой системы. Среди всего множества гамильтоновых систем выделим 
семейство вполне интегрируемых систем. Свойство интегрируемости гамильтоновой системы зависит лишь от свойств движения как такового, а не от того или иного выбора переменных $\mathcal{M}$. Таким образом, любые канонические преобразования координат фазового пространства $\mathcal{M}$ сохраняют свойство интегрируемости.

Для расширенного фазового пространства $\mathcal{M}_{E}$ ситуация сложнее, т.к. отсутствует регулярный способ нахождения канонических преобразований, отображаюших данную вполне интегрируемую систему в другую интегрируемую систему.Каждое найденное преобразование приводит либо к новой интегрируемой модели, либо устанавливает связи между известными интегрируемыми моделями, считавшимися до этого совершенно разными.

Рассмотрим вполне интегрируемую систему с гамильтонианом $H$, которая имеет $n$ функционально независимых интегралов $\left\{I_{j}\right\}_{j=1}^{n}$ в инволюции

$$
\left\{I_{j}, I_{k}\right\}=0, \quad j, k=1, \ldots, n, \quad I_{1}=H .
$$

По теореме Лиувилля, если поверхность уровня интегралов движения компактна, то в ее окрестности можно ввести канонические координаты действие-угол $s_{1}, \ldots, s_{n}$ и $\varphi_{1}, \ldots, \varphi_{n}$ так, что

$$
\Omega=\sum_{j=1}^{n} s_{j} \wedge \varphi_{j}
$$

В переменных действие-угол преобразование (2.2) изменяет только половину уравнений движения

$$
\frac{d \varphi_{j}}{d t}=\omega_{j}\left(s_{1}, \ldots, s_{n}\right) \mapsto \frac{d \varphi_{j}}{d \tilde{t}}=\frac{\omega_{j}\left(s_{1}, \ldots, s_{n}\right)}{v(t, s, \varphi)},
$$

не изменяя остальных уравнений движения,

$$
\frac{d s_{j}}{d t}=0 \mapsto \frac{d s_{j}}{d \tilde{t}}=0
$$

Вследствие этого, для того чтобы преобразование времени (2.2) было каноническим, достаточно, чтобы функция $v$ являлась функцией только от половины $\left\{s_{j}\right\}_{j=1}^{n}$ переменных фазового пространства $\mathcal{M}$. В этом случае исходная функция Гамильтона факторизуется

$$
H(s)=v(s) \widetilde{H}(s)
$$

на общей поверхности уровня $\mathcal{M}_{\alpha}$. С помощью данного разложения ниже будет построено некоторое обобщение систем типа Штеккеля.

Однако, если нас интересует задача явного построения канонических преобразований времени, сохраняюших интегрируемость для данной гамильтоновой системы, то, к сожалению, мы не всегда можем воспользоваться теоремой Лиувилля и переменными действие-угол. Более того, даже используя переменные действие-угол, не всегда можно построить новое семейство интегралов движения для гамильтониана $\widetilde{H}(s)$. Иногда удобнее использовать представление уравнений движения вполне интегрируемых систем в форме Лакса $\{H, L\}=[L, A][1,5]$, так что коэффициенты характеристического 
полинома матрицы $L$ порождают интегралы движения в инволюции. В этом подходе вопрос о построении канонического преобразования (2.2), (2.3), сохраняюшего свойство интегрируемости, можно свести к изучению алгебраических преобразований матриц Лакса и геометрических преобразований соответствующих спектральных алгебраических кривых.

Рассмотрим в качестве примера сохраняющее интегрируемость преобразование времени в задаче Кеплера. По-видимому, впервые такое преобразование использовалось Кеплером для решения уравнений движения. Изучение преобразования соответствующих функций Гамильтона и остальных свойств замены времени Кеплера было продолжено в работах $[6,7]$.

Итак, рассмотрим двумерный осциллятор с гамильтонианом

$$
H=H_{0}+a V(q)=\left(p_{1}^{2}+p_{2}^{2}+b\right)+a\left(q_{1}^{2}+q_{2}^{2}\right) .
$$

После канонического преобразования (2.3) с функцией

$$
v(p, q)=V(q)=q_{1}^{2}+q_{2}^{2}
$$

и точечного преобразования переменных $\left(p_{1}, q_{1}, p_{2}, q_{2}\right) \rightarrow\left(p_{x}, x, p_{y}, y\right)$ гамильтониан двумерного осциллятора отображается в гамильтониан задачи Кеплера

$$
\widetilde{H}=\frac{H_{0}(p, q)}{v(q)}+a=p_{x}^{2}+p_{y}^{2}+\frac{b}{\sqrt{x^{2}+y^{2}}}+a .
$$

Данные системы являются вырожденными системами типа Штеккеля. Подобное преобразование времени может быть построено для любой из систем Штеккеля [8]. Преобразование вида (2.7) порождает для некоторых из них следующее отображение матриц Лакса:

$$
L(\lambda) \mapsto \tilde{L}(\lambda)=L(\lambda)+\left(\begin{array}{cc}
0 & 0 \\
\widetilde{H} & 0
\end{array}\right),
$$

а для других систем выглядит более сложно [8]. Далее мы докажем, что преобразование матриц Лакса вида (2.8) может быть использовано для цепочек Тоды и систем Холта, которые не принадлежат семейству штеккелевских систем.

Для систем типа Штеккеля преобразование соответствуюших спектральных кривых $\mathcal{C}: \operatorname{det}(L(\lambda)+\mu I)=0$ можно представить в следующем виде:

$$
\begin{array}{ll}
\mathcal{C}: & \mu^{2}=\sum_{j \neq m \neq k}^{g} a_{j} \lambda^{j}+a_{m} \lambda^{m}+H \lambda^{k}, \\
\widetilde{\mathcal{C}}: & \mu^{2}=\sum_{j \neq m \neq k}^{g} a_{j} \lambda^{j}+\widetilde{H} \lambda^{m}+a_{k} \lambda^{k} .
\end{array}
$$

Здесь $n$ коэффициентов $a_{j}$ являются интегралами движения $J_{j}$, так что $a_{k}=H$. Остальные $g-n$ коэффициентов - параметры (заряды), которые определяют потенциальную часть функции Гамильтона $H=T(p)+V(q, a)$. Преобразование времени приводит 
к перестановке местами коэффициента $a_{m}=H$ (функции Гамильтона) и одного из параметров $a_{k}$ (заряда). Напомним, что в квантовой механике от задачи на собственные значения для исходного гамильтониана $H$ :

$$
H(p, q) \Psi=\left(H_{0}+a V+b\right) \Psi=E \Psi
$$

который представим в виде суммы свободного гамильтониана $H_{0}$ и взаимодействия с некоторым параметром $a$, можно перейти к задаче на собственные значения константы связи $а$ (заряда)

$$
\widetilde{H}(p, q) \widetilde{\Psi}=\left(\widetilde{H}_{0}+(b-E) V^{-1}\right) \widetilde{\Psi}=-a \widetilde{\Psi} .
$$

Например, при помоши такого преобразования можно получить собственные значения энергии и собственные функции атома водорода из соответствующих величин для осциллятора и наоборот. Изучение подобных преобразований и их свойств в квантовой механике началось с работ Фока [9] и Шредингера [10] по кулоновской задаче и продолжается до настояшего времени [11]. В русской литературе преобразования такого типа иногда называют штурмовскими. Таким образом, замену времени в классической механике (2.2) можно рассматривать как аналог штурмовского преобразования в квантовой механике. При этом в методе Бирмана-Швингера функция $v(q)=V(q)$ называется “сэндвич”-потенциалом [11]. Заметим, что математический аппарат штурмовских преобразований в настоящее время более развит, чем теория соответствующих канонических преобразований времени в классической механике.

\section{3. ПРЕОБРАЗОВАНИЕ ВРЕМЕНИ КЕПЛЕРОВСКОГО ТИПА}

В данном разделе построены канонические преобразования времени (2.2), которые можно называть кеплеровскими или штурмовскими преобразованиями, т.к. они индуцируют преобразование матриц Лакса вида (2.8) и преобразование спектральных кривых вида (2.9).

3.1. Цепочки Тоды. Поскольку цепочки Тоды будут использоваться для построения новых интегрируемых систем, мы начнем с краткого описания этих цепочек. Всю необходимую информацию о цепочках Тоды можно найти в обзорах $[1,5]$.

Пусть $\mathfrak{g}-$ простая алгебра Ли ранга $n=\operatorname{rank} \mathfrak{g}$ с естественной градуировкой, $\mathfrak{a}-\mathrm{ee}$ подалгебра Картана, $K$ - форма Киллинга, $P$ - система простых корней. Для корня $\alpha \in P$ обозначим через $\mathfrak{g}_{\alpha}$ соответствующее корневое пространство и через $e_{\alpha} \in \mathfrak{g}_{\alpha}-$ корневой вектор, нормированный так, что $K\left(e_{\alpha}, e_{-\alpha}\right)=1$. Корневое разложение $\mathfrak{g}=$ $\mathfrak{a}+\bigoplus_{\alpha \in P} \mathfrak{g}_{\alpha}$ порождает естественную градуировку высотой корня. Построим обобшенное разложение Гаусса $\mathfrak{g}=\mathfrak{g}_{+}+\mathfrak{g}_{-}$, вводя борелевскую подалгебру $\mathfrak{g}_{+}=\bigoplus_{i \geqslant 0} \mathfrak{g}_{i}$ и соответствуюшую нильпотентную подалгебру $\mathfrak{g}_{-}=\bigoplus_{i<0} \mathfrak{g}_{i}$. Данное разложение порождает дуальное разложение $\mathfrak{g}^{*}=\mathfrak{g}_{+}^{*}+\mathfrak{g}_{-}^{*}$ и вложение $i=\mathfrak{g}_{+}^{*} \hookrightarrow \mathfrak{g}^{*}$.

Как обычно, пространство $\mathfrak{g}^{*}$ будем отождествлять с $\mathfrak{g}$ при помощи формы Киллинга $K$. Выберем вектор вида

$$
a=\sum_{\alpha \in P} a_{\alpha} e_{-\alpha}, \quad a_{\alpha} \in \mathbb{R}
$$


и рассмотрим орбиту $\mathcal{O}_{a}$ точки $a$ относительно подалгебры $\mathfrak{g}_{+}$в $\mathfrak{a}+\mathfrak{g}_{-1}$. Орбита $\mathcal{O}_{a}$ состоит из точек

$$
\xi=p+\sum_{\alpha \in P} c_{\alpha} a_{\alpha} e_{-\alpha}, \quad p \in \mathfrak{a}, \quad c_{\alpha}>0
$$

Легко указать канонические переменные, отождествляюшие $\mathcal{O}_{a}$ с пространством $T^{*} \mathfrak{a}$. Пусть $\left\{h_{j}\right\}$ - базис в $\mathfrak{a},\left\{h_{j}^{\prime}\right\}$ - двойственный базис относительно формы Киллинга $K\left(h_{i}, h_{j}^{\prime}\right)=\delta_{i j}$. Определим канонические переменные следуюшим образом:

$$
\begin{gathered}
q_{j}=K\left(q, h_{j}\right), \quad p_{j}=K\left(p, h_{j}^{\prime}\right), \quad p, q \in \mathfrak{a} \\
\left\{p_{i}, p_{j}\right\}=\left\{q_{i}, q_{j}\right\}=0, \quad\left\{p_{i}, q_{j}\right\}=\delta_{i j}
\end{gathered}
$$

Орбита $\mathcal{O}_{a}$ параметризуется данными переменными так:

$$
\xi=\sum_{i=1}^{n} p_{i} h_{i}+\sum_{\alpha \in P} a_{\alpha}\left[\exp \left(\sum_{i=1}^{n} q_{i} K\left(\alpha, h_{i}^{\prime}\right)\right)\right] e_{-\alpha} .
$$

Ограничение инвариантных функций $\phi$ в $\mathfrak{g}$ на данную орбиту приводит к слишком простой динамике. Поэтому добавим к орбите $\mathcal{O}_{a}$ вектор $e=\sum_{\alpha \in P} e_{\alpha}$ из подалгебры $\mathfrak{g}_{+}$, который можно рассматривать как произвольную постоянную одноточечную $\mathfrak{g}_{-}$-орбиту. Получаюшаяся орбита

$$
\mathcal{O}_{a e}=\mathcal{O}_{a}+e
$$

в алгебре $\mathfrak{g}_{R}=\mathfrak{g}_{+} \oplus \mathfrak{g}_{-}$состоит из точек вида

$$
L=\sum_{i=1}^{n} p_{i} h_{i}+\sum_{\alpha \in P} a_{\alpha}[\exp K(\alpha, q)] e_{-\alpha}+\sum_{\alpha \in P} e_{\alpha} .
$$

Ограничение квадратичной функции Казимира

$$
H(X)=\frac{1}{2} K(X, X)
$$

на орбиту $\mathcal{O}_{a e}(3.1)$ порождает функцию Гамильтона для обобщенных цепочек Тоды

$$
H(p, q)=\frac{1}{2} K(p, p)+\sum_{\alpha \in P} a_{\alpha} e^{\alpha(q)} .
$$

Для данной орбиты выпишем одно из представлений Лакса [1]

$$
\{H, L\}=[L, A], \quad A=-\sum_{\alpha \in P} a_{\alpha}[\exp K(\alpha, q)] e_{-\alpha} .
$$

Теперь от исходного фазового пространства $\mathcal{M}$ перейдем к расширенному $\mathcal{M}_{E}$. Используя метод Адлера-Костанта, добавим к исходной динамической орбите $\mathcal{O}_{a}$ постоянную орбиту е из оставшейся части расширенного пространства $\mathcal{M}_{E}(2.8)$. Как обычно, элемент е должен быть постоянным относительно нового времени, принадлежать остатку пространства $\mathcal{M}_{E}$ и должен аннулировать $\left[\mathfrak{g}_{ \pm}, \mathfrak{g}_{ \pm}\right]$, т.е. являться характером $\mathfrak{g}_{ \pm}^{*}$. Более того, потребуем, чтобы ограничение исходной квадратичной функции Казимира (3.3) порождало константу связи $K(\tilde{L}, \tilde{L})=-b$ вместо гамильтониана $\widetilde{H}$ по аналогии с системами типа Штеккеля (2.9) [8]. 
ПРЕДЛОЖЕНИЕ 1. Для любого простого корня $\beta \in P$ и любого числа $b_{\beta} \in \mathbb{R}$ функиия Гамильтона вида

$$
\widetilde{H}_{\beta}(p, q)=e^{-\beta(q)}\left(H(p, q)+b_{\beta}\right)
$$

имеет $(n-1)$ функционально независимых дополнительных интегралов движсения, которые порождаются инвариантами матрицы $\tilde{L}_{\beta}$ вида

$$
\tilde{L}_{\beta}=L-\widetilde{H}_{\beta} \frac{e_{\beta}}{a_{\beta}} .
$$

Соответствующее представление Лакса равно

$$
\{\tilde{H}, \tilde{L}\}=[\tilde{L}, \tilde{A}], \quad \tilde{A}=e^{-\beta(q)} A .
$$

Здесь $H, L$ и A- соответственно функиия Гамильтона (3.4) и матриць Лакса (3.2), (3.5) для обобщенных иепочек Тоды.

Из уравнения (3.8) следует, что инварианты $\tilde{L}$ являются интегралами движения. Подсчет числа интегралов и доказательство их функциональной независимости могут быть проведены по аналогии с цепочками Тоды.

Обобшенные периодические цепочки Тоды, связанные с аффинными алгебрами Ли $\mathcal{L}(\mathfrak{g})$, также можно использовать для построения новых интегрируемых систем вида (3.6). При этом не все гамильтонианы $\widetilde{H}_{\beta}$, отвечающие корням $\beta \in P$, являются функционально независимыми. Число функционально независимых гамильтонианов $H_{\beta}, \quad \beta \in P$, определяется симметрией соответствующей корневой системы.

Теперь кратко рассмотрим несколько вспомогательных соображений об алгебраической природе преобразования матриц Лакса вида (3.8). Напомним, что одним из ключевых элементов в обшей схеме является корневое разложение $\mathfrak{g}=\mathfrak{a}+\oplus \mathfrak{g}_{\alpha}$, которое порождает естественную градуировку высотой корня. Данная градуировка определяет также все возможные вложения трехмерной подалгебры $A_{1} \simeq s l(2)$ в алгебру $\mathfrak{g}$. Пусть $\left\{\mathbf{e}_{-}, \mathbf{e}_{+}, \mathbf{h}\right\}-$ базис в алгебре $s l(2)$ :

$$
\left[\mathbf{h}, \mathbf{e}_{-}\right]=\mathbf{e}_{-}, \quad\left[\mathbf{h}, \mathbf{e}_{+}\right]=-\mathbf{e}_{+}, \quad\left[\mathbf{e}_{-}, \mathbf{e}_{+}\right]=2 \mathbf{h} .
$$

Элемент

$$
\boldsymbol{\Delta}=\mathbf{h}^{2}+\frac{1}{2}\left(\mathbf{e}_{-} \mathbf{e}_{+}+\mathbf{e}_{+} \mathbf{e}_{-}\right)
$$

обертываюшей алгебры является оператором Лапласа на группе $S L(2)$. Рассмотрим бесконечномерное представление $\mathcal{W}$ алгебры $s l(2)$ в линейном пространстве $V$ так, что

$$
\mathcal{W}:\left\{\mathbf{e}_{-}, \mathbf{e}_{+}, \mathbf{h}\right\} \rightarrow\left\{e_{-}, e_{+}, h\right\} \in \operatorname{End}(V) .
$$

Если оператор $е$ обратим в пространстве $\operatorname{End}(V)$, то для любой функции $\varphi(\Delta)$ от значения оператора Казимира (3.10) отображение

$$
e_{-} \rightarrow e_{-}^{\prime}=e_{-}, \quad h \rightarrow h^{\prime}=h, \quad e_{+} \rightarrow e_{+}^{\prime}=e_{+}+e_{-}^{-1} \varphi(\Delta)
$$


является внешним автоморфизмом пространства бесконечномерных представлений алгебры $s l(2)$ в пространстве $V$ [12]. Данное отображение сдвигает спектр оператора Лапласа (3.10) по правилу

$$
\Delta \rightarrow \Delta^{\prime}=\Delta+\varphi(\Delta)
$$

В частности, при $\varphi(\Delta)=-(\Delta+b)$ получим $\Delta \rightarrow \Delta^{\prime}=-b$. Таким образом, используя внешний автоморфизм (3.11), от рассмотрения спектра $\Delta$ оператора Лапласа (3.10) на группе мы переходим к спектру некоторой “константы связи” $b$, что можно рассматривать как алгебраический аналог штурмовского преобразования.

Рассмотрим теперь цепочку Тоды, отвечающую произвольной простой алгебре Ли $\mathfrak{g}$, которая содержит подалгебру $A_{1}$, некоторым образом в нее вложенную. Преобразование орбит (3.8) порождается преобразованием (3.11), если вместо значения квадратичного оператора Казимира (3.3), ограниченного на подалгебру $A_{1}$ и равного $\Delta$ из (3.10), подставить ограничение данного оператора Казимира на орбиту $\mathcal{O}_{a e}$, равное $H$ из (3.4). Заметим, что автоморфизм (3.11) нетривиально действует только на одну из нильпотентных подалгебр $s l(2)$. Расширение данного отображения на орбиту $\mathcal{O}_{a e}$ будет действовать нетривиально именно на ту нильпотентную подалгебру, в которой орбита $\mathcal{O}_{a e}$ параметризуется постоянным вектором.

Обсудим подробнее периодические цепочки Тоды, связанные с алгебрами Ли серии $A_{n}$. Рассмотрим представление, в котором $(n \times n)$-матрицы Лакса имеют следуюший вид:

$$
\begin{aligned}
L(\mu) & =\sum_{i=1}^{n} p_{j} E_{i, i}+\sum_{i=1}^{n-1}\left(a_{i} e^{q_{i}-q_{i+1}} E_{i+1, i}+E_{i, i+1}\right)+\frac{a_{n} e^{q_{n}-q_{1}}}{\mu} E_{1, n}+\mu E_{n, 1} \\
A(\mu) & =\sum_{i=1}^{n-1} a_{i} e^{q_{i}-q_{i+1}} E_{i+1, i}+\frac{a_{n} e^{q_{n}-q_{1}}}{\mu} E_{1, n} .
\end{aligned}
$$

Здесь $E_{j, k}$ - матрица, элемент которой, стоящий в $j$-й строке и $k$-м столбце, равен единице, остальные элементы равны нулю. Соответствующая функция Гамильтона (3.4) есть

$$
H=\frac{1}{2} \operatorname{tr} L(\mu)=\frac{1}{2} \sum_{i=1}^{n} p_{i}^{2}+a_{i} e^{q_{i}-q_{i+1}} .
$$

Замена времени $(2.3),(3.6)$, отвечающая корню $\beta=\varepsilon_{j}-\varepsilon_{j+1}$, имеет вид

$$
v(q)=e^{-\beta(q)}=\exp \left(q_{j+1}-q_{j}\right), \quad \widetilde{H}=e^{q_{j+1}-q_{j}}(H+b) .
$$

Она индуцирует преобразование матриц Лакса

$$
\tilde{L}=L-\frac{\widetilde{H}}{a_{j}} E_{j, j+1}, \quad \tilde{A}=v^{-1} A
$$


и преобразование спектральных кривых $\operatorname{det}(L(\mu)+\lambda I)=0$ вида

$$
\begin{array}{ll}
\mathcal{C}: & -\frac{\prod_{i=1}^{n} a_{i}}{\mu}-\mu=\lambda^{n}+\lambda^{n-1} P+\lambda^{n-2}\left(\frac{P^{2}}{2}-H\right)+\cdots, \\
\widetilde{\mathcal{C}}: & -\frac{\prod_{i=1}^{n} a_{i}}{\mu}-\mu\left(1-\frac{\widetilde{H}}{a_{j}}\right)=\lambda^{n}+\lambda^{n-1} P+\lambda^{n-2}\left(\frac{P^{2}}{2}+b\right)+\cdots .
\end{array}
$$

Здесь $P=\sum p_{j}$ - полный импульс системы, $H$ и $\widetilde{H}$ - функции Гамильтона. Сравнивая кривые $\mathcal{C}$ и $\widetilde{\mathcal{C}}$, видим, что коэффициент при $\lambda^{n-2}$, который прежде зависел от гамильтониана $H$, теперь зависит от константы связи $b$, что опять позволяет говорить об аналогии со штурмовским преобразованием в квантовой механике. В то же время новый гамильтониан $\widetilde{H}$ входит теперь коэффициентом при втором спектральном параметре $\mu$.

Для краткости положим далее все параметры $a_{i}=1$ и запишем скобки Пуассона между матрицами Лакса в обычном $r$-матричном виде

$$
\{\stackrel{1}{L}(\mu), \stackrel{2}{L}(\nu)\}=\left[r_{12}(\mu, \nu), \stackrel{1}{L}(\mu)\right]+\left[r_{21}(\mu, \nu) \stackrel{2}{L}(\nu)\right]
$$

где использованы стандартные обозначения

$$
\stackrel{1}{L}(\mu)=L(\mu) \otimes I, \quad \stackrel{2}{L}(\nu)=I \otimes L(\nu), \quad r_{21}(\mu, \nu)=-\Pi r_{12}(\nu, \mu) \Pi
$$

$\Pi$ - оператор перестановки во вспомогательном пространстве $\mathbb{C}^{n} \times \mathbb{C}^{n}[13]$. Замена времени (2.2), (3.14) переводит постоянную $r$-матрицу для цепочек Тоды

$$
r_{12}(\mu, \nu)=r_{12}^{\mathrm{const}}(\mu, \nu)=\frac{1}{\mu-\nu}\left(\nu \sum_{m \geqslant i}+\mu \sum_{m<i}\right) E_{i m} \otimes E_{m i}
$$

в динамическую

$$
r_{12}(\mu, \nu)=r_{12}^{\mathrm{const}}(\mu, \nu)+r_{12}^{\mathrm{dyn}}(\mu, \nu), \quad r_{12}^{\mathrm{dyn}}(\mu, \nu)=\tilde{A}(\nu, q) \otimes E_{j, j+1} .
$$

Вторая матрица в уравнении Лакса $\tilde{A}(\nu, q)$ и соответственно динамическая $r$-матрица зависят только от координат.

Рассмотрим теперь преобразование Беклунда $B_{\lambda}$ для цепочек Тоды [14]. Напомним, что $B_{\lambda}$ является каноническим преобразованием $(p, q) \mapsto(P, Q)$ исходного фазового пространства $\mathcal{M}$, которое оставляет инвариантными интегралы движения $I_{j}(p, q)=$ $I_{j}(P, Q)$. Следуя работе [14], определим $B_{\lambda}$ для цепочек Тоды, используя производящую функцию вида

$$
F_{\lambda}(q \mid Q)=\sum_{i=1}^{n}\left(e^{q_{i}-Q_{i}}-e^{Q_{i}-q_{i+1}}-\lambda\left(q_{i}-Q_{i}\right)\right)
$$

так что

$$
p_{i}=\frac{\partial F}{\partial q_{i}}, \quad P_{i}=-\frac{\partial F}{\partial Q_{i}}
$$


Инвариантность интегралов движения $I_{k}(P, Q)=J_{k}(p, q)$ доказывается при помоши сплетаюшего соотношения

$$
M(\mu, q, Q) L(\mu, p, q)=L(\mu, P, Q) M(\mu, q, Q)
$$

с матрищей

$$
M(\mu, q, Q)=\sum_{i=1}^{n-1} e^{Q_{i}-q_{i+1}} E_{i+1, i}+\frac{a_{n} e^{Q_{n}-q_{1}}}{\mu} E_{1, n} .
$$

Замена времени (2.2), (3.14) приводит к сдвигу производяшей функции по правилу

$$
\begin{aligned}
\widetilde{F}_{\lambda}(q \mid Q) & =\sum_{i=1}^{n}\left(e^{q_{i}-Q_{i}}-e^{Q_{i}-q_{i+1}}\left(1-\delta_{i j} \widetilde{H}\right)-\lambda\left(q_{i}-Q_{i}\right)\right)= \\
& =F_{\lambda}(q, Q)+\widetilde{H} e^{Q_{j}-q_{j+1}}=F_{\lambda}(q, Q)+\Delta F
\end{aligned}
$$

В данном случае $\widetilde{H}$ является элементом расширенного фазового пространства, а не функцией от координат $\{p, q\}$. Это означает, что при вычислении импульсов $p$ и $P$ в соотношениях (3.18) необходимо положить все частные производные от $\widetilde{H}$ равными нулю. Сплетаюшее соотношение (3.19) и матрица $M(\mu, q, Q)(3.20)$ остаются при этом неизменными.

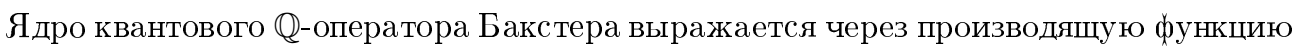
$F_{\lambda}(q \mid Q)$ преобразования Беклунда [15]. Таким образом, замена времени приводит к факторизации $\mathbb{Q}$-оператора в квазиклассическом пределе

$$
\widetilde{\mathbb{Q}} \sim \exp \left(-\frac{i \widetilde{F}}{\hbar}\right)=\mathbb{Q} \exp \left(-\frac{i \Delta F}{\hbar}\right) .
$$

Напомним, что $\mathbb{Q}$-оператор является производящей функцией квантовых интегралов движения. Поэтому, зная квазиклассический спектр квантовых интегралов одной системы, мы можем пытаться исследовать соответствуюший спектр для другой системы, используя сдвиг (3.21) и перестановку интегралов (3.16). Заметим, что подобная процедура используется в методе Бирмана-Швингера в рамках совершенно иного математического аппарата [11].

Известно, что для цепочек Тоды, отвечаюших алгебрам Ли классических бесконечных серий $A_{n}, \ldots, D_{n}$, сушествует не только представление вида $(3.2)$, но и $(2 \times 2)$-матрицы Лакса $[13,16]$. Так, для цепочек, связанных с системой корней $A_{n}$, второе представление Лакса имеет вид

$$
T(u)=T_{1} T_{2} \ldots T_{n}, \quad \text { где } \quad T_{j}=\left(\begin{array}{cc}
u+p_{j} & a_{j} e^{q_{j}} \\
-e^{-q_{j}} & 0
\end{array}\right), \quad a_{j} \in \mathbb{R}
$$

так что

$$
\left\{H, T_{j}\right\}=T_{j} A_{j}-A_{j-1} T_{j}, \quad\{H, T\}=\left[T, A_{n}\right]
$$


и

$$
A_{j}=\left(\begin{array}{cc}
u & a_{j} e^{q_{j}} \\
-e^{-q_{j}} & 0
\end{array}\right) .
$$

Замена времени вида (2.2), (3.14) индуцирует следуюшее преобразование матриц Лакca:

$$
\begin{aligned}
& \widetilde{T}=T_{1} \ldots T_{j-1}\left[T_{j} T_{j+1}+\left(\begin{array}{cc}
H+b & 0 \\
0 & 0
\end{array}\right)\right] T_{j+2} \ldots T_{n}, \\
& \tilde{A}_{n}=v^{-1}(q) A_{n},
\end{aligned}
$$

и соответствуюших спектральных данных

$$
\begin{array}{ll}
\operatorname{det} T=\prod_{i=1}^{n} a_{i}, & \operatorname{tr} T=u^{n}+u^{n-1} P+u^{n-2}\left(\frac{P^{2}}{2}-H\right)+\cdots, \\
\operatorname{det} \widetilde{T}=\left(a_{j}-\widetilde{H}\right) \prod_{i \neq j}^{n} a_{i}, & \operatorname{tr} \widetilde{T}=u^{n}+u^{n-1} P+u^{n-2}\left(\frac{P^{2}}{2}+b\right)+\cdots
\end{array}
$$

аналогично преобразованиям для систем типа Штеккеля [8].

Если положить все $a_{j}=1$, то скобки Пуассона между исходными $(2 \times 2)$-матрицами Лакса $T(\lambda)$, определенными в (3.22), представляются в виде квадратичных скобок Склянина $[13,16]$

$$
\left\{\frac{1}{T}\left(u_{1}\right), \stackrel{2}{T}\left(u_{2}\right)\right\}=\left[R\left(u_{1}-u_{2}\right), \stackrel{1}{T}\left(u_{1}\right) \stackrel{2}{T}\left(u_{2}\right)\right], \quad R\left(u_{1}-u_{2}\right)=\frac{\Pi}{u_{1}-u_{2}} .
$$

Замена времени (3.14) отображает скобки Склянина в квадратично-линейные скобки

$$
\left\{\stackrel{\frac{1}{T}}{\widetilde{T}}\left(u_{1}\right), \stackrel{2}{\widetilde{T}}\left(u_{2}\right)\right\}=\left[R\left(u_{1}-u_{2}\right), \stackrel{\frac{1}{\widetilde{T}}}{\left(u_{1}\right)} \stackrel{2}{\widetilde{T}}\left(u_{2}\right)\right]+\left[r_{12}^{\mathrm{dyn}}\left(u_{1}, u_{2}\right), \stackrel{\frac{1}{T}}{T}\left(u_{1}\right)\right]+\left[r_{21}^{\mathrm{dyn}}\left(u_{1}, u_{2}\right), \stackrel{2}{\widetilde{T}}\left(u_{2}\right)\right]
$$

с динамическими $r$-матрицами вида

$$
r_{12}^{\mathrm{dyn}}\left(u_{1}, u_{2}\right)=A_{n}\left(u_{1}, q\right) \otimes\left(T_{1} \ldots T_{j-1}\left(\begin{array}{ll}
1 & 0 \\
0 & 0
\end{array}\right) T_{j+1} \otimes T_{n}\right) .
$$

Здесь все матрицы $T_{k}$ зависят от спектрального параметра $u_{2}$, а $A_{n}\left(u_{1}, q\right)$ - вторая матрица в уравнении Лакса (3.23).

По-прежнему преобразования Беклунда $B_{\lambda}(3.17)$ и $\widetilde{B}_{\lambda}$ (3.21) являются изоспектральными преобразованиями матриц монодромии $T$ и $\widetilde{T}$. В первом случае это можно доказать, используя сплетаюшие соотношения [15]

$$
M_{i}(u, \lambda) T_{i}(p, q)=T_{i}(P, Q) M_{i+1}(u, l)
$$

с матрицей

$$
M_{i}=\left(\begin{array}{cc}
1 & e^{Q_{i-1}} \\
-e^{-q_{i}} & \lambda-u-e^{Q_{i-1}-q_{i}}
\end{array}\right) .
$$


При $i \neq j, j+1$ данные соотношения справедливы и после замены времени. Добавочное соотношение во втором случае имеет вид

$$
\begin{aligned}
& M_{j}(u, \lambda)\left[T_{j}(p, q) T_{j+1}(p, q)+\left(\begin{array}{cc}
H+b & 0 \\
0 & 0
\end{array}\right)\right]= \\
& \quad=\left[T_{j}(P, Q) T_{j+1}(P, Q)+\left(\begin{array}{cc}
H+b & 0 \\
0 & 0
\end{array}\right)\right] M_{j+2}(u, \lambda)
\end{aligned}
$$

и не факторизуется на множители.

Обсудим теперь вопрос о канонических преобразованиях, которые позволяют переписать полученную функцию Гамильтона $\widetilde{H}(2.3)$ в натуральном виде. Общего решения данной задачи нам не известно. Поэтому ограничимся примером трехчастичной цепочки Тоды. С помошью интеграла движения $P$ (импульс центра масс) можно свести гамильтонову систему (3.13) при $n=3$ с тремя степенями свободы к системе с двумя степенями свободы, т.е. редуцировать фазовое пространство от 6-мерного до 4-мерного. После редукции и точечного преобразования координат по правилу

$$
\begin{aligned}
q_{1}= & \frac{1}{2}(1+i \sqrt{3}) \ln x+\frac{1}{2}(1-i \sqrt{3}) \ln y, \\
p_{1}= & \frac{1}{2}\left(1-\frac{i}{\sqrt{3}}\right) x p_{x}+\frac{1}{2}\left(1+\frac{i}{\sqrt{3}}\right) y p_{y}, \\
q_{2}= & \frac{1}{2}(-1+i \sqrt{3}) \ln x-\frac{1}{2}(1+i \sqrt{3}) \ln y, \\
p_{2}= & \frac{1}{2}\left(-1-\frac{i}{\sqrt{3}}\right) x p_{x}-\frac{1}{2}\left(1-\frac{i}{\sqrt{3}}\right) y p_{y}
\end{aligned}
$$

гамильтониан $\widetilde{H}$ приобретает вид

$$
\widetilde{H}=p_{x} p_{y}+\frac{b}{x y}+a_{2} x^{z_{1}} y^{z_{2}}+a_{3} x^{z_{2}} y^{z_{1}}+a_{1}
$$

где $z_{j}$ - корни уравнения $\left(z-z_{1}\right)\left(z-z_{2}\right)=z^{2}+3 z+3=0$.

Данная система с гамильтонианом (3.26) и с кубическим по импульсам дополнительным интегралом $K$ была найдена в работе [17] еще до открытия соответствуюшей цепочки Тоды. Все остальные девять интегрируемых систем, найденных в работе [17], также связаны преобразованиями вида (2.3) с известными интегрируемыми системами. Более подробно данный вопрос будет рассмотрен в последующих публикациях.

В заключение этого пункта выпишем двухчастичные функции Гамильтона для нескольких аффинных алгебр типа $X_{2}^{(1)}$. После подходящей точечной замены координат типа (3.25) соответствующие гамильтонианы имеют вид

$$
\widetilde{H}=p_{x} p_{y}+\frac{b}{x y}+a x^{z_{1}} y^{z_{2}}+c x^{s_{1}} y^{s_{2}}+d, \quad a, b, c, d \in \mathbb{R}
$$


где показатели степеней $z_{1,2}$ и $s_{1,2}$ являются корнями различных алгебраических уравнений второго порядка. Далее для каждой из алгебр мы выпишем только соответствующие пары уравнений:

$$
\begin{array}{llrl}
A_{3}^{(1)}: & z^{2}+3 z+3=0, & s^{2}+3 s+3=0 ; \\
B_{2}^{(1)} C_{2}^{(1)}: & z^{2}+4 z+5=0, & s^{2}+4 s+5=0, \\
z^{2}+2 z+2=0, & s^{2}+3 s+\frac{5}{2}=0 ; \\
D_{2}^{(1)}: & z^{2}+2 z+2=0, & s^{2}+2 s+2=0, \\
G_{2}^{(1)}: & z^{2}+2 z+2=0, & (s+2)^{2}=0 ; \\
z^{2}+2 z+4=0, & s^{2}+3 s+3=0, \\
z^{2}+3 z+\frac{7}{3}=0, & s^{2}+3 s+3=0 .
\end{array}
$$

При этом дополнительные интегралы движения $K$ являются соответственно полиномами третьей, четвертой и шестой степеней по импульсам. Заметим, что для алгебры типа $A_{3}^{(1)}$ все три гамильтониана $H_{\beta}, \beta \in P$, равны, для алгебр типа $B_{2}^{(1)}, C_{2}^{(1)}$ и $D_{2}^{(1)}$ два гамильтониана независимы, а для алгебры типа $G_{2}^{(1)}$ все три гамильтониана различны.

3.2. Системы типа Холта. Функция Гамильтона для систем типа Холта имеет вид

$$
\widetilde{H}=\frac{1}{2}\left(p_{X}^{2}+p_{Y}^{2}\right)+a X^{-\frac{2}{3}}\left(\frac{3 b}{4} X^{2}+Y^{2}+c\right) .
$$

Известно только три интегрируемых системы данного типа $[18,19]$ :

$$
b=1, \quad b=6, \quad b=16 .
$$

Остальные параметры $a$ и $c$ во всех трех случаях произвольны. Переопределив параметры $a$ и $c$ :

$$
a \rightarrow 4\left(\frac{3}{2}\right)^{\frac{1}{3}} a, \quad c \rightarrow \frac{c}{3 a},
$$

и используя точечное преобразование

$$
\begin{array}{ll}
X=\frac{2}{3} x^{\frac{3}{2}}, & p_{X}=p_{x} \sqrt{x} \\
Y=-\frac{1}{2 \sqrt{3 a}} p_{y}, & p_{Y}=2 \sqrt{3 a} y
\end{array}
$$

перепишем функцию Гамильтона (3.27) в виде

$$
\widetilde{H}=\frac{p_{x}^{2}+p_{y}^{2}}{2 x}+2 a\left(b x^{2}+3 y^{2}\right)+\frac{2 c}{x} .
$$


Можно утверждать, что каноническое преобразование времени $(2.2),(2.3)$ при $v=x$ переводит системы типа Холта в системы Энона-Эйлеса, сохраняя свойство интегрируемости. Напомним, что функция Гамильтона для систем типа Энона-Эйлеса имеет вид

$$
H=\frac{p_{x}^{2}+p_{y}^{2}}{2}+2 a x\left(b x^{2}+3 y^{2}\right)+2 c=x \widetilde{H} .
$$

Замена времени и преобразование матриц Лакса для случая $b=6$ рассматривались в статье [8]. Поэтому этот случай далее не рассматривается.

Для случая $b=1$ матришы Лакса можно найти, например, в работе [20]. Они имеют вид

$$
L(\lambda)=\left(\begin{array}{ccc}
6 x \lambda & -\gamma\left(3 x^{2}+y^{2}\right) & \frac{3}{2 \gamma}\left(3 \lambda^{2}-p_{x}\right) \\
\frac{3}{2 \gamma}\left(3 \lambda^{2}+p_{x}\right) & -3 x \lambda-\frac{p_{y} y}{\lambda} & \frac{y^{2}}{\lambda} \\
-\gamma\left(3 x^{2}+y^{2}\right) & 9 \lambda^{3}-\frac{y^{2}}{\lambda} & -3 x \lambda+\frac{p_{y} y}{\lambda}
\end{array}\right)
$$

$$
A(\lambda)=\left(\begin{array}{ccc}
0 & 2 \gamma \lambda & 0 \\
0 & 0 & 1 \\
2 \gamma \lambda & -4 \gamma^{2} x & 0
\end{array}\right), \quad \text { где } \quad \gamma=\sqrt{3 a} .
$$

Как и для цепочек Тоды, каноническое преобразование времени $(2.2),(3.29)$ при $v=x$ можно называть кеплеровским или штурмовским преобразованием, т.к. оно индуцирует подобное преобразование матриц Лакса (2.8), (3.15)

$$
\tilde{L}(\lambda)=L(\lambda)+\frac{3}{2 \gamma} \widetilde{H}\left(\begin{array}{lll}
0 & 1 & 0 \\
0 & 0 & 0 \\
1 & 0 & 0
\end{array}\right), \quad \tilde{A}(\lambda)=v^{-1} A(\lambda)
$$

и соответствуюших спектральных кривых

$$
\begin{array}{ll}
\mathcal{C}: & -12 a \mu^{3}=729 \lambda^{7}-162 H \lambda^{3}+324 c \lambda^{3}+\frac{K^{2}}{\lambda}, \\
\widetilde{\mathcal{C}}: & -12 a \mu^{3}+54 \widetilde{H} \mu \lambda^{2}=729 \lambda^{7}+324 c \lambda^{3}+\frac{\widetilde{K}^{2}}{\lambda},
\end{array}
$$

где $K$ и $\widetilde{K}$-вторые интегралы движения.

Для случая $b=16$ матрицы Лакса имеют вид [20]

$$
\begin{aligned}
& L(\lambda)=\left(\begin{array}{ccc}
12 x-\frac{p_{y} y}{2 \lambda} & \frac{y^{2}}{4 \lambda} & \frac{3}{8 a} \\
9 \lambda+3 p_{x}+\frac{6 a x y^{2}}{\lambda}-\frac{p_{y}^{2}}{2 \lambda} & -6 x & \frac{y^{2}}{4 \lambda} \\
-24 a\left(\frac{24 x^{2}+y^{2}}{2}-\frac{x y p_{y}}{\lambda}\right) & 9 \lambda-3 p_{x}-\frac{6 a x y^{2}}{\lambda}-\frac{p_{y}^{2}}{2 \lambda} & -6 x+\frac{p_{y} y}{2 \lambda}
\end{array}\right), \\
& A(\lambda)=\left(\begin{array}{ccc}
0 & 1 & 0 \\
0 & 0 & 1 \\
24 a\left(\lambda-p_{x}\right) & -48 a x & 0
\end{array}\right) .
\end{aligned}
$$


Как и для цепочек Тоды, каноническая замена времени (2.2) при $v=x$ индуцирует аналогичное преобразование матриц Лакса

$$
\tilde{L}(\lambda)=L(\lambda)+3 \widetilde{H}\left(\begin{array}{lll}
0 & 0 & 0 \\
0 & 0 & 0 \\
1 & 0 & 0
\end{array}\right), \quad \tilde{A}(\lambda)=v^{-1} A(\lambda)
$$

и соответствующих спектральных кривых

$$
\begin{array}{ll}
\mathcal{C}: & -8 a \mu^{3}=243 \lambda^{2}-54 H+108 c+\frac{3 K}{\lambda^{2}}, \\
\widetilde{\mathcal{C}}: & -8 a \mu^{3}+9 \widetilde{H} \mu=243 \lambda^{2}+108 c+\frac{3 \widetilde{K}}{\lambda^{2}} .
\end{array}
$$

Таким образом, мы получили ранее неизвестное представление Лакса для системы типа Холта при $b=1$ и $b=16$.

\section{4. СИСТЕМЫ ТИПА ШТЕККЕЛЯ}

Метод разделения переменных является одним из основных методов интегрирования уравнений движения динамических систем. Данный метод позволяет свести интегрирование для случая многих степеней свободы к интегрированию последовательности одномерных задач. В работах Якоби [21] было предложено рассматривать обратную задачу, т.е. начиная с некоторого набора разделенных одномерных задач построить различные классы вполне интегрируемых систем. При этом заданные переменные разделения отображаются различными способами в физические переменные, что и определяет различные методы разделения переменных. Одними из простейших систем, построенных в рамках этого подхода, являются системы типа Лиувилля и типа Штеккеля. В данном разделе предложено обобшение семейства штеккелевских систем.

На заданном фазовом пространстве $\mathcal{M}$ рассмотрим две интегрируемые системы, которые определяются интегралами движения $\left\{I_{j}\right\}_{j=1}^{n}$ и $\left\{J_{j}\right\}_{j=1}^{n}$, функционально независимы и находятся в инволюции

$$
\left\{I_{j}, I_{k}\right\}=0, \quad\left\{J_{j}, J_{k}\right\}=0, \quad j, k=1, \ldots, n .
$$

По теореме Лиувилля для каждой из данных систем можно ввести две системы переменных действие-угол $\left\{s_{j}, \varphi_{j}\right\}_{j=1}^{n}$ и $\left\{\tilde{s}_{j}, \tilde{\varphi}_{j}\right\}_{j=1}^{n}$. При этом функции Гамильтона зависят только от переменных действия $H=I_{1}\left(s_{1}, \ldots, s_{n}\right), \widetilde{H}=J_{1}\left(\tilde{s}_{1}, \ldots, \tilde{s}_{n}\right)$. Если эти две различные системы координат связаны:

$$
\tilde{s}_{j}=\tilde{s}_{j}\left(s_{1}, \ldots, s_{n}\right),
$$

то в соответствии с разложением (2.5) преобразование расширенного фазового пространства (2.2) при

$$
v(p, q)=v(s)=I_{1}(p, q) \quad \text { или } \quad v(p, q)=v(s)=J_{2}(p, q)
$$

является каноническим и отображает данную пару интегрируемых систем в третью интегрируемую систему.

Для построения данного отображения мы должны, во-первых, проверить выполнение условия (4.1), используя исходные физические переменные, и, во-вторых, явно построить новое семейство интегралов движения. 
ПРЕДЛОЖЕНИЕ 2. Если все разности интегралов движения $\left(I_{j}-J_{j}\right), \quad j=$ $1, \ldots, n$, находятся в инволючии друг с другом:

$$
\left\{I_{j}-J_{j}, I_{k}-J_{k}\right\}=0, \quad j, k=1, \ldots, n,
$$

то отношение любъх двух интегралов

$$
K_{m}=\frac{I_{m}}{J_{m}}
$$

и функиии $K_{j}(j \neq m)$ от исходных интегралов движсения (число әтих функиий равно $n-1)$ :

$$
K_{j}=\frac{(\mathbf{I} \wedge \mathbf{J})_{m j}}{J_{m}}=\frac{I_{m} J_{j}-I_{j} J_{m}}{J_{m}}=K_{m} J_{j}-I_{j}, \quad m \neq j=1, \ldots, n
$$

также являются интегралами движения, которые определяют новую интегри руемую систему на фазовом пространстве $\mathbb{R}^{2 n}$.

Здесь мы использовали $m$-й столбец внешнего произведения $(\mathbf{I} \wedge \mathbf{J})$ двух независимых векторов $\mathbf{I}$ и $\mathbf{J}$ в пространстве $\mathbb{R}^{n}$ для определения функций $K_{j}$. Заметим, что в данном предложении не требуется функциональная независимость всех разностей интегралов $\left(I_{j}-J_{j}\right)$. Достаточно потребовать, чтобы два вектора $\mathbf{I}$ и $\mathbf{J}$ в пространстве $\mathbb{R}^{n}$ были независимы, тогда и $n$ функций $K_{m}$ и $K_{j}$ будут функционально независимыми. В силу теоремы Лиувилля для доказательства интегрируемости новой системы остается доказать, что все величины $K_{m}$ и $K_{j}$ находятся в инволюции. Вычислим скобки между выделенным интегралом $K_{m}(4.3)$ и другими интегралами движения $K_{j}(4.4)$,

$$
\left\{K_{m}, K_{j}\right\}=\frac{I_{m}}{J_{m}^{2}}\left(\left\{I_{m}, J_{j}\right\}+\left\{J_{m}, I_{j}\right\}\right)=-\frac{I_{m}}{J_{m}^{2}}\left\{I_{m}-J_{m}, I_{j}-J_{j}\right\}=0 .
$$

Используя данньй результат, вычислим оставшиеся скобки

$$
\begin{aligned}
\left\{K_{j}, K_{k}\right\} & =K_{m}\left\{J_{j}, K_{k}\right\}-\left\{I_{j}, K_{k}\right\}= \\
& =J_{k}\left(K_{m}\left\{J_{j}, K_{m}\right\}-\left\{I_{j}, K_{m}\right\}\right)-K_{m}\left(\left\{J_{j}, I_{k}\right\}+\left\{I_{j}, J_{k}\right\}\right)= \\
& =J_{k}\left(\left\{K_{j}, K_{m}\right\}+K_{m}\left\{I_{j}-J_{j}, I_{k}-J_{k}\right\}\right)=0, \quad j \neq k \neq m .
\end{aligned}
$$

Таким образом, мы доказали, что преобразование алгебр интегралов движения

$$
(\mathbf{I}, \mathbf{J}) \rightarrow \mathbf{K}
$$

вида (4.3), (4.4) сохраняет свойство интегрируемости. Можно рассматривать данное преобразование как каноническое преобразование (2.3) интеграла $I_{m}$ при $v(p, q)=J_{m}$. Чтобы использовать это преобразование для построения новых интегрируемых систем, необходимо найти исходные интегрируемые системы, удовлетворяюшие условию (4.2). 
В дальнейшем мы докажем, что любые две интегрируемые системы типа Штеккеля [2] с одинаковой матрицей Штеккеля удовлетворяют условию (4.2).

Напомним, что интегрируемые штеккелевские системы $[2,5]$ однозначно определяются невырожденной $(n \times n)$-матрицей Штеккеля $\mathbf{S}$, элементы $j$-го столбца которой $\mathbf{s}_{k j}$ зависят лишь от координаты $q_{j}$,

$$
\operatorname{det} \mathbf{S} \neq 0, \quad \frac{\partial \mathbf{s}_{k j}}{\partial q_{m}}=0, \quad j \neq m .
$$

Используя матрицу $\mathbf{S}$, построим $n$ квадратичных по импульсам функционально независимых интегралов движения $I_{k}, k=1, \ldots, n$, в инволюции по правилу

$$
I_{k}=\sum_{j=1}^{n} c_{j k}\left[p_{j}^{2}+U_{j}\left(q_{j}\right)\right], \quad c_{j k}=\frac{\mathbf{S}_{k j}}{\operatorname{det} \mathbf{S}} .
$$

Здесь $\mathbf{C}=\left[c_{j k}\right]$ - матрица, обратная к матрице Штеккеля $\mathbf{S}, \mathbf{a} \mathbf{S}_{k j}$ - алгебраическое дополнение элемента $\mathbf{s}_{k j}$ в определителе матрищы $\mathbf{S}$. Как видим, алгебра $\mathcal{A}_{I}$ штеккелевских интегралов движения (4.6) имеет простейшую градуировку по степеням импульсов. При этом и коэффициенты при квадратах импульсов, и свободные члены определяются матрицей Штеккеля $\mathbf{S}$. Такая градуировка достаточно бедна, и поэтому далее мы будем использовать более широкую алгебру интегралов движения для штеккелевских систем.

Напомним, что основной проблемой подхода Штеккеля к построению интегрируемых систем является переход от переменных разделения к физическим переменным, например к таким переменным $\left\{p_{j}, x_{j}\right\}_{j=1}^{n}$, в терминах которых гамильтониан приобретает натуральный вид и уравнения движения становятся уравнениями Ньютона. Частично данная проблема решена для штеккелевских систем с одинаковыми потенциалами вида $U_{j}=U, j=1, \ldots, n$, при введении пары Лакса [22].

В соответствии с работой [8] все множество штеккелевских систем в $\mathbb{R}^{2 n}$ можно разбить на семейства дуальных систем, связанных преобразованием. Действительно, любые две матрицы Штеккеля $\mathbf{S}$ и $\widetilde{\mathbf{S}}$, которые отличаются друг от друга только одной $m$-й строкой

$$
\mathbf{s}_{k j}=\tilde{\mathbf{s}}_{k j}, \quad k \neq m,
$$

определяют дуальные интегрируемые системы с гамильтонианами $I_{m}$ и $\tilde{I}_{m}$,

$$
\tilde{I}_{m} \longleftrightarrow I_{m}, \quad \tilde{I}_{m}=\frac{I_{m}(p, q)}{v(q)}
$$

для которых замена времени [23] задается отношением определителей

$$
v\left(q_{1}, \ldots, q_{n}\right)=\frac{\operatorname{det} \mathbf{S}\left(q_{1}, \ldots, q_{n}\right)}{\operatorname{det} \widetilde{\mathbf{S}}\left(q_{1}, \ldots, q_{n}\right)}
$$

данных матриц Штеккеля. Преобразование (4.7) связывает интегрируемые системы с двумя разными матрицами Штеккеля. 
Рассмотрим теперь две интегрируемые системы типа Штеккеля с общей матрицей $\mathbf{S}$. Пусть интегралы движения для первой системы имеют вид $\left\{I_{k}\right\}$ (4.6), а вторая система обладает семейством интегралов вида

$$
J_{k}=\sum_{j=1}^{n} c_{j k}\left[p_{j}^{2}+W_{j}\left(q_{j}\right)\right], \quad k=1, \ldots, n .
$$

Здесь хотя бы один из потенциалов $U_{j}\left(q_{j}\right)$ первой системы функционально не зависим от соответствуюшего потенциала $W_{j}\left(q_{j}\right)$ второй системы.

ПРЕДЛОЖЕНИЕ 3. Любъе две интегрируемые системы типа Штеккеля (4.6) с общей матрицей $\mathbf{S}$ и функиионально независимьми потенииалами $U_{j}\left(q_{j}\right)$ u $W_{j}\left(q_{j}\right)$ удовлетворяют условиям (4.2). Таким образом, отношение функиий Гамильтона типа Штеккеля определяет новую интегрируемую систему.

$$
K_{m} \longleftrightarrow\left(I_{m}, J_{m}\right), \quad K_{m}=\frac{I_{m}(p, q)}{J_{m}(p, q)} .
$$

Действительно, для двух штеккелевских систем (4.6) и (4.8) с разными потеншиалами $U_{j}$ и $W_{j}$ все разности интегралов движения $I_{k}$ и $J_{k}$ зависят только от половины координат $\left\{q_{j}\right\}_{j=1}^{n}$ фазового пространства $\mathbb{R}^{2 n}$ :

$$
\left(I_{k}-J_{k}\right)=\sum_{j=1}^{2} c_{j k}\left[U_{j}\left(q_{j}\right)-W_{j}\left(q_{j}\right)\right] .
$$

Таким образом, все скобки Пуассона между ними равны нулю и системы с обшей матрицей Штеккеля удовлетворяют условию (4.2).

Хотя новые интегралы движения $K_{j}(4.3),(4.4)$ не являются квадратичными функциями от импульсов, тем не менее их специальный вид позволяет свести интегрирование уравнений движения к квадратурам.

ПРЕДЛОЖЕНИЕ 4. Для гамильтоновой системь с функиией Гамильтона

$$
H=K_{m}=\frac{\sum_{j=1}^{n} c_{j m}\left[p_{j}^{2}+U_{j}\left(q_{j}\right)\right]}{\sum_{j=1}^{n} c_{j m}\left[p_{j}^{2}+W_{j}\left(q_{j}\right)\right]}
$$

следующие утверждения әквивалентны:

а) соответствующие уравнения Гамильтона-Якоби допускают разделение переменных;

б) существует невырожденная порядка п матрица Штеккеля $\mathbf{S}$, әлемент $\mathbf{s}_{k j}$ которой зависит лишь от $q_{j}$, причем выполнень условия (4.5);

в) система с гамильтонианом (4.10) обладает $(n-1)$ функиионально независимыми интегралами движения вида (4.4).

Доказать данное предложение можно, повторяя доказательство аналогичного утверждения для систем типа Штеккеля $[5,24]$ и используя первые два предложения данного раздела. 
Здесь мы рассмотрим только вопрос о разделении переменных и об интегрировании уравнений движения в квадратурах. Отметим, что совместная поверхность уровня всех интегралов (4.3)

$$
\mathcal{M}_{a}=\left\{z \in \mathbb{R}^{2 n}: K_{j}(z)=\alpha_{j}, j=1, \ldots, n\right\}
$$

диффеоморфна $n$-мерному тору, как и для обычных систем типа Штеккеля. Действительно, введем обозначение

$$
V_{j}\left(q_{j}\right)=\left(1-\alpha_{m}\right)^{-1}\left(U_{j}\left(q_{j}\right)-\alpha_{m} W_{j}\left(q_{j}\right)\right)
$$

Используя определения (4.6), (4.8) и (4.10), получим следующие равенства:

$$
\begin{aligned}
& \sum_{j=1}^{n} c_{j m}\left[p_{j}^{2}+V_{j}\left(q_{j}\right)\right]=0=\beta_{m}, \\
& \sum_{j=1}^{n} c_{j k}\left[p_{j}^{2}+V_{j}\left(q_{j}\right)\right]=-\frac{\alpha_{k}}{1-\alpha_{m}}=\beta_{k}, \quad m \neq k=1, \ldots, n .
\end{aligned}
$$

Умножая данные равенства на матрищу Штеккеля, получим

$$
p_{j}^{2}=\left(\frac{\partial \mathcal{S}}{\partial q_{j}}\right)^{2}=\sum_{k=1}^{n} \beta_{k} \mathbf{s}_{k j}\left(q_{j}\right)-V_{j}\left(q_{j}\right)
$$

где $\mathcal{S}\left(q_{1}, \ldots, q_{n}\right)$ - функция укороченного действия. Поэтому для рассматриваемого случая уравнение Гамильтона-Якоби на $M_{\alpha}(4.11)$

$$
\frac{\partial \mathcal{S}}{\partial t}+H\left(t, \frac{\partial \mathcal{S}}{\partial q_{1}}, \ldots, \frac{\partial \mathcal{S}}{\partial q_{n}}, q_{1}, \ldots, q_{n}\right)=0 \quad \Rightarrow \quad c_{j m} \frac{\partial \mathcal{S}}{\partial q_{j}} \frac{\partial \mathcal{S}}{\partial q_{j}}=E
$$

допускает разделение переменных

$$
\mathcal{S}\left(q_{1}, \ldots, q_{n}\right)=\sum_{j=1}^{n} \mathcal{S}_{j}\left(q_{j}\right)
$$

где функция $\mathcal{S}_{j}\left(q_{j}\right)$ задается формулой

$$
\mathcal{S}_{j}\left(q_{j}\right)=\int \sqrt{\sum_{k=1}^{n} \beta_{k} \mathbf{s}_{k j}\left(q_{j}\right)-V_{j}\left(q_{j}\right)} d q_{j}
$$

Согласно стандартной процедуре интегрирования уравнения Гамильтона-Якоби величины $q_{j}\left(t, \alpha_{1}, \ldots, \alpha_{n}\right)$ определяются из уравнений

$$
\sum_{j=1}^{n} \int \frac{\mathbf{s}_{k j}(\lambda) d \lambda}{\sqrt{\sum_{k=1}^{n} \beta_{k} \mathbf{s}_{k j}(\lambda)-V_{j}(\lambda)}}=\delta_{k}, \quad k=1, \ldots, n
$$


где константы $\alpha_{j}$ и $\beta_{j}$ связаны преобразованием (4.12). Заметим, что, сдвигая интеграл $I_{m}$ на произвольное число, мы можем снять ограничение $\beta_{m}=0$.

Таким образом, задача интегрирования уравнений движения для системы (4.9) сводится к стандартной проблеме алгебраической геометрии - задаче обрашения отображения Абеля. Напомним, что преобразование времени (4.7) может быть связано с неоднозначностью данного отображения Абеля. Алгебро-геометрическая природа нового преобразования (4.9) пока не выяснена.

Для штеккелевских систем с одинаковыми потенциалами вида $U_{j}=U, j=1, \ldots, n$, известны $(2 \times 2)$-матрицы Лакса [22]. Замена времени (4.7) индуцирует преобразование матриц Лакса, которое, как и для цепочек Тоды, является сдвигом на постоянную относительно нового времени матришу, принадлежашую расширенному фазовому пространству [8]. Соответствуюшее новому преобразованию времени (4.9) преобразование матриц Лакса будет рассмотрено в отдельной работе.

Конечно, новая функшия Гамильтона $H=K_{m}(4.10)$ выглядит достаточно сложно. Однако можно использовать канонические замены координат, для того чтобы переписать ее в более простом виде. На нескольких примерах мы покажем, что после ряда подходяших замен переменных новый гамильтониан $H$ можно представить в натуральном виде $H=T+V$ как сумму кинетической и потенциальной энергий. Напомним, что даже для квадратичных по импульсам штеккелевских гамильтонианов (4.6) данная задача решена только частично.

В качестве примеров рассмотрим системы с двумя степенями свободы, которые допускают разделение переменных в полярных координатах. Для определенности будем использовать стандартные обозначения координат $\left(p_{r}, r\right)$ и $\left(p_{\phi}, \phi\right)$ вместо $\left(p_{1}, q_{1}\right)$ и $\left(p_{2}, q_{2}\right)$ соответственно.

Ограничимся простейшим случаем плоского движения, который описывает движение в центральном поле с интегралами вида

$$
I_{1}=p_{r}^{2}+\frac{p_{\phi}^{2}}{r^{2}}-a^{2} r^{2 k}+b, \quad I_{2}=p_{\phi}, \quad a, b, n \in \mathbb{R} .
$$

В качестве второй системы рассмотрим систему, отвечаюшую свободному движению,

$$
J_{1}=p_{r}^{2}+\frac{p_{\phi}^{2}}{r^{2}}, \quad J_{2}=p_{\phi} .
$$

Все гамильтонианы натурального вида с рациональньми потенциалами, допускаюшие точечное разделение переменных в стандартных криволинейных координатах, принадлежат классу однородных систем типа Штеккеля. Поэтому обе эти системы являются системами типа Штеккеля с общей матрицей Штеккеля вида

$$
\mathbf{S}=\left(\begin{array}{cc}
1 & 0 \\
-r^{-2} & 1
\end{array}\right)
$$

В данном случае $\mathbf{s}_{12}=c_{21}=0$ и $U(\phi)=W(\phi)$, откуда следует, что $I_{2}=J_{2}$. В силу этого одна из разностей интегралов тождественно равна нулю, $\left(I_{2}-J_{2}\right)=0$, и мы можем 
использовать вторые интегралы $I_{2}=J_{2}$ в произвольной форме $\phi\left(I_{2}\right)=\phi\left(J_{2}\right)$, отличной от штеккелевской квадратичной (4.6). Например, в случаях (4.13) и (4.14) мы использовали корень из штеккелевского интеграла, что отвечает выбору другого базиса в абелевой подалгебре алгебры $\mathcal{A}_{I J}$ интегралов движения.

Покажем, что отношение (4.3) функций Гамильтона (4.13), (4.14) можно переписать в натуральном виде после ряда канонических преобразований координат. Сначала перейдем от криволинейных координат к декартовым:

$$
\begin{aligned}
r & =\sqrt{u v}, & \phi & =i \operatorname{arctg}\left(\frac{u-v}{u+v}\right), \\
p_{r} & =-\frac{u p_{u}+v p_{v}}{r}, & p_{\phi} & =i\left(u p_{u}-v p_{v}\right) .
\end{aligned}
$$

Затем переставим местами координаты и импульсы $\left(u \leftrightarrow p_{u}\right),\left(v \leftrightarrow p_{v}\right)$ так, чтобы новый гамильтониан стал полиномом по импульсам:

$$
H=K_{1}=\frac{I_{1}}{J_{1}}=-\frac{a^{2}}{4} \frac{p_{u}^{k} p_{v}^{k}}{u v}+\frac{b}{4 u v}+1
$$

В заключение применим точечное преобразование

$$
\begin{aligned}
& p_{x}=p_{u} u^{\frac{1}{k+1}}, \quad x=\left(1+\frac{1}{k}\right) u^{\frac{k}{k+1}}, \\
& p_{y}=p_{v} v^{\frac{1}{k+1}}, \quad y=\left(1+\frac{1}{k}\right) v^{\frac{k}{k+1}},
\end{aligned}
$$

которое позволяет разделить гамильтониан (4.16) на кинетическую и потенциальную части:

$$
H=T+V=p_{x}^{k} p_{y}^{k}+a(x y)^{-\frac{k}{k+1}}+b,
$$

после масштабирования $H \rightarrow \gamma H$ и переопределения параметров $a$ и $b$ в потеншиале.

Матришы Штеккеля определены на половине фазового пространства $\mathbb{R}^{2 n}$ и зависят только от координат $q_{j}$. Таким образом, остается некоторая дополнительная свобода, связанная с каноническими преобразованиями подпространства импульсов $p_{j}$ вида

$$
\begin{gathered}
\left(p_{1}, \ldots, p_{n}\right) \rightarrow\left(\tilde{p}_{1}, \ldots, \tilde{p}_{n}\right), \\
p_{i}-\tilde{p}_{i}=2 \frac{\partial F\left(q_{1}, \ldots, q_{n}\right)}{\partial q_{i}}, \quad i=1, \ldots, n .
\end{gathered}
$$

Здесь $F\left(q_{1}, \ldots, q_{n}\right)$ - производяшая функция преобразования $(4.17)$, заданная как функция от координат $\left\{q_{j}\right\}$, которые не изменяются при данном преобразовании.

Если условие (4.2) инвариантно относительно данных преобразований, то можно использовать эти преобразования для построения новых интегрируемых систем (4.3). В обшем случае класс канонических преобразований вида (4.17) слишком широк для исследования, и поэтому здесь мы ограничимся только одним содержательным примером. 
Как и в предыдущем случае, рассмотрим движение в центральном поле с интегралами $I_{1,2}(4.13)$ и свободное движение с интегралами движения вида

$$
J_{1}=\tilde{p}_{r}^{2}+\frac{\tilde{p}_{\phi}^{2}}{r^{2}}, \quad J_{2}=\tilde{p}_{\phi}
$$

Здесь новые импульсы $\left(\tilde{p}_{r}, \tilde{p}_{\phi}\right)$ связаны со старыми импульсами $\left(p_{r}, p_{\phi}\right)$ следующим каноническим преобразованием типа (4.17):

$$
\tilde{p}_{r}=p_{r}-\frac{\partial f(r)}{\partial r} \frac{\cos (n \phi)}{n}, \quad \tilde{p}_{\phi}=p_{\phi}+f(r) \sin (n \phi) .
$$

Функция $f(r)$ и константа $n$ пока произвольны.

Обе эти системы принадлежат штеккелевскому семейству систем. Однако в отличие от предыдущего примера $\left(I_{2}-J_{2}\right) \neq 0$. Если вторые интегралы движения взять в квадратичной штеккелевской форме (4.6), то условие (4.2) не инвариантно относительно канонических преобразований вида (4.19). Поэтому в качестве второго интеграла мы преднамеренно используем корень из квадратичного штеккелевского интеграла (4.6). Только в этом случае обе интегрируемые системы (4.13) и (4.18) удовлетворяют условию (4.2) при следующем ограничении на функцию $f(r)(4.19)$ :

$$
\frac{f^{\prime}(r)}{f(r)}=\frac{n}{r} \Rightarrow f(r)=c r^{n}, \quad c \in \mathbb{R} .
$$

Перейдем теперь к декартовым координатам по правилу (4.15) для координат и следующему правилу для импульсов:

$$
\tilde{p}_{r}=-\frac{u p_{u}+v p_{v}}{r}, \quad \tilde{p}_{\phi}=i\left(u p_{u}-v p_{v}\right) .
$$

Далее опять используем перестановку координат и импульсов $\left(u \leftrightarrow p_{u}\right),\left(v \leftrightarrow p_{v}\right)$ так, чтобы новьй гамильтониан стал полиномом по импульсам:

$$
H=\frac{I_{1}}{J_{1}}=\frac{1}{4 u v}\left(c^{2} p_{u}^{n-1} p_{v}^{n-1}-a^{2} p_{u}^{k} p_{v}^{k}-2 c\left(v p_{u}^{n-1}+u p_{v}^{n-1}\right)\right)+\frac{b}{4 u v}+1 .
$$

Очевидно, что при $c=0$ мы получим систему (4.16). В другом предельном случае $a=c$, $k=n-1$ после точечного канонического преобразования (4.17) функция Гамильтона (4.21) в новых переменных принимает вид

$$
H=p_{x}^{k}+p_{y}^{k}+a(x y)^{-\frac{k}{k+1}}+b .
$$

Здесь мы опять использовали масштабирование и переопределили параметры потенциала. При $k=2$ гамильтониан $H=K_{1}$ будет квадратичным полиномом по импульсам, а второй дополнительньй интеграл $K_{2}(4.4)$ кубичен по импульсам [25].

Таким образом, в качестве примера мы построили семейство интегрируемых систем на плоскости, которое содержит в себе и задачу Кеплера, и систему Фокаса-Лагерстрема при некоторых специальных значениях параметров.

Благодарности. Автору приятно поблагодарить И. В. Комарова за многочисленные полезные обсуждения и интерес к работе, а также рецензента за сделанные замечания. Работа выполнена при финансовой поддержке Минобразования РФ, грант № 97-40-1.14-25, и гранта РФФИ № 99-01-00698. 


\section{Список литературы}

[1] А.Г. Рейман, М.А. Семенов-Тян-Шанский. Теоретико-групповые методы в теории интегрируемых систем. В сб.: Итоги науки и техники. Современные проблемы математики. Фундаментальные направления. Т. 16. Динамические системы-7. Ред. Р. В. Гамкрелидзе. М.: ВИНИТИ, 1987. С. 119.

[2] P. Stäckel. Compt. Rend. Acad. Sci. (Paris). 1893. V. 116. P. 485; P. 1284.

[3] К. Ланцош. Вариационные принципы механики. М.: Мир, 1965.

[4] Дж. Л. Синг. Классическая динамика. М.: Физматгиз, 1963.

[5] А. М. Переломов. Интегрируемые системы классической механики и алгебры Ли. М.: Наука, 1990.

[6] T. Levi-Civita. Acta Math. 1906. V. 30. P. 305.

[7] J. Moser. Commun. Pure Appl. Math. 1970. V. 23. P. 609.

[8] А. В. Цызанов. ТМФ. 1999. Т. 120. С. 27.

[9] V.A. Fock. Z. Physik. 1935. V. 98. P. 145.

[10] E. Schrödinger. Proc. Roy. Irish. Acad. A. 1940. V. 46. P. 9; P. 183; 1941. V. 47. P. 53.

[11] М. Рид, Б. Саймон. Методы современной математической физики. Т. 3. М.: Мир, 1982.

[12] А. В. Цицанов. ТМФ. 1999. Т. 118. С. 205.

[13] Л.Д. Фаддеев, Л.А. Тахтаджяян. Гамильтонов подход в теории солитонов. М.: Наука, 1986.

[14] H. Flaschka, D. Mc.Laughlin. In: Bäcklund Transformations. Lect. Notes Math. V. 515. Ed. R. Miura. Berlin: Springer, 1976. P. 225.

[15] E. K. Sklyanin, V. B. Kuznetsov. J. Phys. A. 1998. V. 31. P. 2241.

[16] В. Б. Кузнецов, А. В Цыъганов. Зап. научн. семин. ЛОМИ. 1989. Т. 172. С. 88.

[17] J. Drach. Compt. Rend. Acad. Sci. (Paris). 1935. V. 200. P. 22.

[18] C. R. Holt. J. Math. Phys. 1982. V. 23. P. 37.

[19] A. Ramani, B. Grammaticos, T. Bountis. Phys. Rep. 1989. V. 180. P. 159.

[20] M. Blaszak, S. Rauch-Wojciechowski. J. Math. Phys. 1994. V. 35. P. 1693.

[21] К. Якоби. Лекции по динамике. М.-Л.: Глав. ред. обшетехнической литературы, 1936.

[22] А. В. Цызанов. ТМФ. 1998. Т. 115. С. 3.

[23] J. Hietarinta, B. Grammaticos, B. Dorizzi, A. Ramani. Phys. Rev. Lett. 1984. V. 53. P. 1707.

[24] L. Pars. Am. Math. Monthly. 1949. V. 56. P. 395.

[25] A.S. Fokas, P. Lagerstrom. J. Math. Anal. Appl. 1980. V. 74. P. 325.

Поступила в редакцию 29.VI.1999 г., после доработки 7.Х.1999 г. 